\title{
Lipid homeostasis and the formation of macrophage-derived foam cells in atherosclerosis
}

\author{
Yuan Yuan ${ }^{1}$, Peng Li ${ }^{2}$, Jing Ye ${ }^{1 凶}$ \\ ${ }^{1}$ Department of Pathology, Xijing Hospital, Fourth Military Medical University, Xi'an 710032, China \\ 2 Tsinghua-Peking Center for Life Sciences, School of Life Sciences, Tsinghua University, Beijing 100084, China \\ $\triangle$ Correspondence: yejing@fmmu.edu.cn \\ Received December 26, 2011 Accepted February 4, 2012
}

\begin{abstract}
Atherosclerosis is a chronic, inflammatory disorder characterized by the deposition of excess lipids in the arterial intima. The formation of macrophage-derived foam cells in a plaque is a hallmark of the development of atherosclerosis. Lipid homeostasis, especially cholesterol homeostasis, plays a crucial role during the formation of foam cells. Recently, lipid droplet-associated proteins, including PAT and CIDE family proteins, have been shown to control the development of atherosclerosis by regulating the formation, growth, stabilization and functions of lipid droplets in macrophage-derived foam cells. This review focuses on the potential mechanisms of formation of macrophage-derived foam cells in atherosclerosis with particular emphasis on the role of lipid homeostasis and lipid droplet-associated proteins. Understanding the process of foam cell formation will aid in the future discovery of novel therapeutic interventions for atherosclerosis.
\end{abstract}

KEYWORDS macrophage, foam cell, atherosclerosis, cholesterol, lipid droplet-associated proteins

\section{INTRODUCTION}

Atherosclerosis, a disease characterized by the deposition of excess lipids in the arterial vessels, has become the leading cause of death and disability in developed countries. The rupture of atherosclerotic plaque walls can cause sudden death by blocking blood supply to the heart or the brain. It is estimated that in the next decade, atherosclerosis and asso- ciated cardiovascular diseases will be a major cause of health and socio-economic problems world-wide. Therefore, it is of significant concern that the factors contributing to atherosclerosis and its progression are still not well defined.

It is generally believed that atherosclerosis is not only a metabolic disease but also an inflammatory disease. The characteristic atherosclerotic lesion is the fibroinflammatory lipid plaque. The early lesion of atherosclerosis, called the "fatty streak," is an aggregate of extracellular lipids and macrophages and smooth muscle cells that are attracted to clean the excess lipids in the arterial intima. However, the advanced atherosclerotic lesions are fibrofatty plaques in the intima of arteries and consist of a huge number of macrophages, smooth muscle cells and inflammatory cells. Most macrophages in atherosclerotic plaques are filled with cytosolic lipid droplets (LDs), resulting in a foamy appearance under a microscope, and these cells are called "foam cells" (Glass and Witztum, 2001). The accumulation of excess lipids in the foam cells is mediated by scavenger receptors on the macrophages located in the subendothelial intima. Accumulation of intracellular lipids, especially free cholesterol, results in cytotoxicity, the release of specific cytokines and the triggering of foam cell death. The release of cellular contents from dead foam cells leads to increased macrophage migration to the intima region, initiating a vicious cycle. As this process progresses, the atheromatous cores of the plaques become necrotic and consist of lipids, cholesterol crystals and cell debris (Takahashi et al., 2002). It was recognized more than a decade ago that the formation of foam cells is central to atherosclerotic plaque development (Siegel-Axel et al., 2008). However, the molecular mechanism of foam cell formation has been determined only recently. 
Although smooth muscle cells and other vascular cells can also migrate into the intima of arteries and form foam cells, in this article, we focus on the mechanism of the formation of macrophage-derived foam cells in atherosclerosis, in particular, the role of cholesterol homeostasis and LD-associated proteins in foam cell formation.

\section{LIPID HOMEOSTASIS}

It is generally believed that the development of atherosclerotic foam cells consists of the following few steps: (1) the endothelium is activated after the accumulation of modified lipoproteins in the intima; (2) monocytes are attracted by chemoattractants and migrate into the intima; (3) monocytes differentiate into macrophages and take up modified lipoproteins; (4) excess lipids accumulate in macrophages, forming lipid-laden foam cells and (5) foam cells die and release their contents, thus attracting more macrophages (Fig. 1). Using human and animal models, it has been clearly shown that the key initiation step of macrophage-derived foam cell formation is the migration of blood-borne monocytes into the subendothelial intima and their further differentiation into macrophages.

In the pre-atherosclerotic stage, serum lipids, especially the oxidized lipoproteins, are deposited under the endothelial cells at the sites of arterial branch points and bifurcations, where the laminar flow is usually disturbed. The overlying endothelial cells become activated and secrete chemoattractants, such as CCL2, CXCL1/KC/Gro $\alpha$, CCL5, CXCL4, and CX3CL1. Simultaneously, the expression of adhesion molecules on the endothelial cells increases. Using the chemokine receptors on their cell surface, monocytes are recruited to the atherosclerotic lesions by the chemoattractants, adhere to the lesional endothelial cells via interactions with adhesion molecules, and finally migrate into the subendothelial spaces. Deletion or blockade of the adhesion molecules, such as ICAM-1, VCAM-1, CD18/ $\beta 2$ integrin, and $P$-selectin, has been shown to slow down the development of atherosclerosis (An et al., 2008).

Once the monocytes enter the subendothelial space, they differentiate into macrophages under chronic inflammatory conditions. Previous studies have shown that the number of circulating monocytes has a positive correlation with the atherosclerotic plaque size in mice, and that functional alterations in monocyte/macrophage cells could affect the development of atherogenesis (Combadière et al., 2008). However, it is believed that different macrophage subsets play different roles in the development of atherosclerosis. On the basis of the differential expression of surface markers and chemokine receptors, two very distinct macrophage phenotypes have been identified. These are called classically activated M1 and alternatively activated M2 macrophages (Martinez et al.,

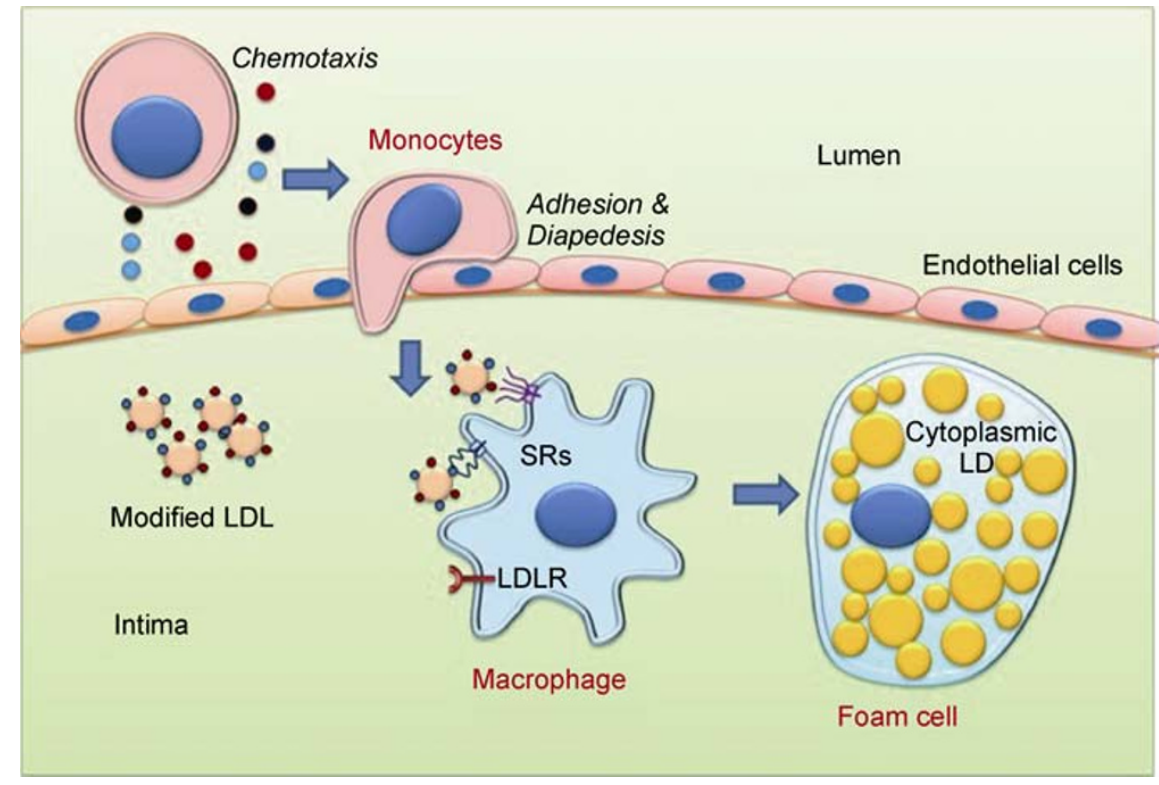

Figure 1. Schematic representation of sequential events involved in the migration of monocytes and their transition into foam cells. Monocytes are critical mediators in all stages of atherosclerosis and are recruited to atherosclerotic lesions by chemoattractants secreted by activated endothelial cells. Simultaneously, the expression of adhesion molecules on the overlying endothelial cells becomes elevated, and monocytes adhere to and roll on the lesional endothelial cells via interactions with these adhesion molecules. When they gain entry into the subendothelial spaces, the infiltrating monocytes transform into macrophages. Monocyte-derived macrophages upregulate the expression of scavenger receptors (SRs), thereby increasing their ability to internalize oxLDL particles and apoptotic cell fragments and become the lipid-laden macrophages called "foam cells." SRs, scavenger receptors; LDLR, LDL receptors; LD, lipid droplet. 


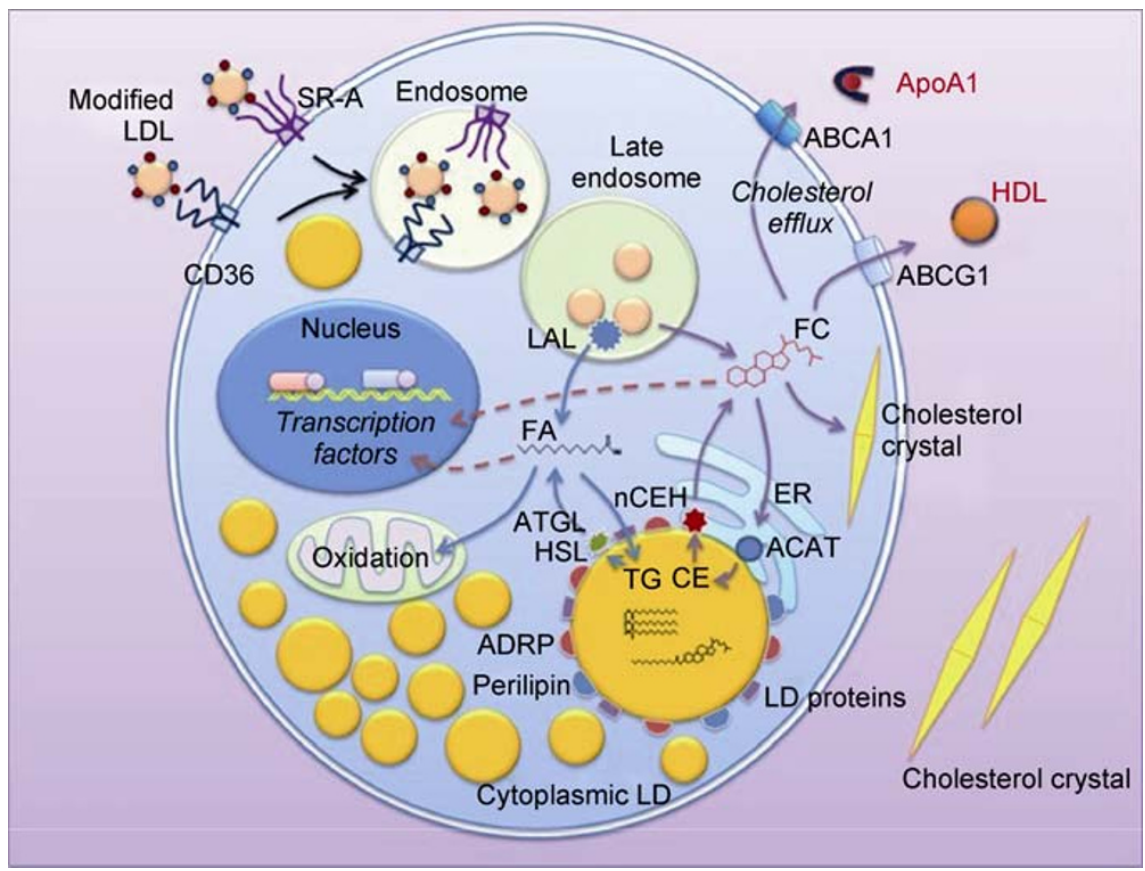

Figure 2. Lipid homeostasis in the process of macrophage-derived foam cell formation. In macrophage-derived foam cells, the excess neutral lipids accumulate in the form of LDs in the cytoplasm. This gives them a foamy appearance and therefore the name "foam cells," which is a hallmark of atherosclerosis. In foam cells, cholesterol homeostasis is maintained by a balance between the influx and efflux pathways. Hydrolysis of CEs represents the first step in FC efflux, and this step is mediated by $\mathrm{nCEH}$, which is recognized as the rate-limiting enzyme. At the same time, triglycerides in internalized lipoproteins are hydrolyzed into glycerol and fatty acids, which are re-esterified into newly formed triglycerides and stored in LDs. Excess FC could be re-esterified by ACAT1 and stored in cytoplasmic LDs. Located on the surfaces of LDs, lipid droplet-associated proteins modulate the formation and catabolism of intracellular LDs. Understanding the characteristics of foam cells and their mechanisms of formation in atherosclerosis is important to better understand lipid homeostasis in foam cells and to discover novel therapeutic interventions to prevent atherosclerosis. ABCA1, ATP-binding cassette transporter A1; ACAT, acyl-CoA:cholesterol acyltransferase; ADRP, adipose differentiation-related protein; ATGL, adipose triglyceride lipase; ApoA1, apolipoprotein A1; CD36, cluster differentiation 36; CE, cholesterol esters; FA, fatty acid; FC, free cholesterol; HDL, high-density lipoprotein; HSL, hormone sensitive lipase; LAL, lysosomal acid lipase; LD, lipid droplet; LDLR, LDL receptors; LXR, liver X receptor; $n C E H$, neutral cholesteryl ester hydrolase; oxLDL, oxidized low-density lipoprotein; PPAR, peroxisome proliferator-activated receptor; SRs, scavenger receptors; TG, triglyceride; SR-A, type A scavenger receptor.

2006). Due to the high levels of SRs, mannose receptors and galactose-type receptors (Martinez et al., 2006) on their surface, M2 macrophages have a high capacity to phagocytose tissue debris and apoptotic bodies and are able to prevent uncontrolled tissue damage (Gordon and Martinez, 2010). Recent studies have described a third macrophage subtype, Mox, which is found in the oxLDL-rich microenvironment of atherosclerosis. These cells develop in response to atherogenic phospholipids and have lower phagocytic and chemotactic capacities than those of M1 and M2 macrophages (Kadl et al., 2010). Much of the current knowledge in this area is based on in vitro studies. Because the situation in atherosclerotic lesions is almost certainly more complex, a clear picture of the heterogeneity of macrophages has not yet emerged from the available studies (Pello et al., 2011).

\section{Lipoprotein uptake}

In the initial atherosclerotic lesion, extracellular modified lipo- proteins are ingested by macrophages by receptor-mediated phagocytosis and pinocytosis. The LDL receptor (LDLR) was thought to play a critical role in the process of modified lipoprotein internalization by macrophages (Zhou et al., 2008). However, macrophage-derived foam cells are found in atherosclerotic lesions even in patients with familial hypercholesterolemia with genetically impaired LDLR. Using acetylated LDL (acLDL) as a model ligand, a new class of receptors (scavenger receptors, SRs) that bind to modified LDLs was identified (Ashraf and Gupta, 2011). Among these SRs, the type A scavenger receptor (SRA) and CD36, a member of the type $B$ family, have been extensively studied (Kunjathoor et al., 2002). It is well known that ApoE-deficient mice, which are widely used as a model of atherosclerosis, develop atherosclerotic lesions throughout the arterial tree (Moore et al., 2005; Manning-Tobin et al., 2009). Interestingly, mice with $C D 36$ and $A p o E$ double deficiency show reduced atherosclerotic lesions and greatly reduced uptake of oxLDL by macrophages compared with their littermate controls 
(Podrez et al., 2000). In contrast to the expression of LDLR, the expression of SRs is not downregulated by increased intracellular levels of cholesterol. As a result, SR-mediated uptake of oxLDL leads to the continuous internalization of lipoproteins and the accumulation of excess quantities of lipoprotein-derived lipids by macrophages (Rios et al., 2011). The excess neutral lipids are accumulated in the form of lipid droplets in the cytoplasm, creating a foamy appearance and giving these macrophages the name "foam cells," a hallmark of atherosclerosis (Fig. 2).

\section{Hydrolysis of cholesterol esters}

Upon internalization, lipoproteins are delivered to the late endosome/lysosome, where cholesterol esters (CEs) are hydrolyzed into free cholesterol (FC) and fatty acids (FAs). Because of the cytotoxicity of FC, the hydrolysis of CEs and the re-esterification of FC are very important in maintaining the functionality of macrophages and foam cells. In lysosomes, lysosomal acid lipase (LAL) hydrolyzes CEs to generate free cholesterol mainly for ABCA1-dependent efflux (Ouimet et al., 2011), whereas the triglycerides (TGs) in internalized lipoproteins are hydrolyzed into glycerol and FAs, which are then re-esterified into newly formed TGs and stored in lipid droplets. As in other cells, FAs in macrophages bind to fatty acid-binding proteins that are responsible for their intracellular trafficking. In macrophages, the absence of aP2, a fatty acid-binding protein, protects ApoE-deficient mice against atherosclerosis (Makowski et al., 2001).

Recently, neutral cholesterol ester hydrolase $(\mathrm{nCEH})$ has been recognized as the rate-limiting enzyme in the hydrolysis of CEs in macrophages (Ghosh et al., 2010). In nCEH transgenic mice crossed into an Ldlr-null background, highfat/cholesterol diet-induced atherosclerosis was significantly reduced because cholesterol efflux and reverse cholesterol transport (RCT) were enhanced, resulting in increased elimination of cholesterol from the body as bile acids in the feces (Zhao et al., 2007). These results demonstrate that macrophage-specific overexpression of $\mathrm{nCEH}$ is antiatherogenic and that this protein is potentially a novel target for the treatment of atherosclerosis.

\section{Re-esterification of free cholesterol}

Upon cholesterol delivery into the endoplasmic reticulum (ER), re-esterification is rapidly stimulated. As in other cell types, it is believed that excess membrane cholesterol and a fraction of LDL-derived FC could be re-esterified by acyl-CoA:cholesterol acyltransferase 1 (ACAT1) and stored in cytoplasmic lipid droplets in foam cells. Inhibition of ACAT activity has been shown to attenuate CE accumulation in foam cells in vitro (Ghosh et al., 2003). However, the selective inhibition of ACAT1 in lesion macrophages in Ldlr-null mice promotes, rather than inhibits, lesion development
(Fazio et al., 2001; Perrey et al., 2001). Although the re-esterification of FC is initially beneficial to the cells, the increase in plaque formation by inhibition of ACAT activity in macrophages correlates with the cytotoxic effects of increased levels of FC, which crystallize in foam cells. The formation of cholesterol crystals, which is easily observed in the lipid-rich necrotic core and often referred to as "the gruel," is indicative of disruption in cholesterol metabolism during the development of atherosclerosis. Moreover, the cholesterol crystals in macrophages promote the maturation and secretion of interleukin-1 $\beta$ (IL-1 $\beta$ ) and IL-18 (Duewell et al., 2010).

\section{Cholesterol efflux}

As previously mentioned, excess FC in foam cells can be re-esterified into CEs to reduce the cytotoxicity. Moreover, it is well established that receptor-mediated FC efflux is another major mechanism for the removal of cellular cholesterol and is critical for preventing the formation of foam cells and the development of atherosclerotic lesions. This process is also thought to be a major mechanism involved in plaque regression when hypercholesterolemia is reversed.

At the plasma membrane, cholesterol is transferred to the outer leaflet. Although passive diffusion plays a minor role, active transport via several transporters is responsible for the bulk of the efflux of cholesterol from macrophages into the serum; this process includes the transport to lipid-poor ApoA1 or HDL by ABCA1 and ABCG1 (Adorni et al., 2007). Although the selective deletion of ABCA1 in macrophages (Brunham et al., 2009) and the overexpression of ABCG1 (Burgess et al., 2008) did not significantly modulate the development of atherosclerotic plaques, simultaneous deficiency in both $A B C A 1$ and $A B C G 1$ promoted atherosclerosis in mice (Yvan-Charvet et al., 2007). In addition to ABCA1 and ABCG1, SR-BI, another type B scavenger receptor, protects against atherosclerosis when expressed in macrophages by stimulating cholesterol efflux, whereas genetically suppressing SR-BI activity in ApoE-deficient mice dramatically accelerates the onset of atherosclerosis (Trigatti et al., 1999). The SR-BI-mediated reverse cholesterol transport process demonstrated in macrophages was confirmed in vivo using ABCA1/SR-BI double knockout mice (Zhao et al., 2011).

\section{REGULATION OF LIPID HOMEOSTASIS}

Macrophage-derived foam cells accumulate a mass of LDs in the cytoplasm as a result of the phagocytosis of matrix-retained lipoproteins and the fluid-phase pinocytosis of non-retained native LDL. Because severe lipotoxicity can be triggered by high intracellular concentrations of lipids, especially FC and FAs (Borradaile et al., 2006), cellular lipid homeostasis is strictly regulated through lipid homeostasis-related transcription factors, such as sterol regulatory element-binding proteins (SREBPs) and peroxisome prolif- 
erator-activated receptors (PPARs).

\section{SREBPs}

SREBPs are transcription factors of the basic-helix-loophelix-leucine zipper (bHLH-Zip) family and exist three isoforms, SREBP-1a, SREBP-1c and SREBP-2 (Brown and Goldstein, 1997). SREBPs play a central role in cellular lipid homeostasis and regulate many genes encoding enzymes involved in cholesterol homeostasis and fatty acid biosynthesis (Brown and Goldstein, 1999). At normal levels, SREBP-1C favors the fatty acid biosynthetic pathway and SREBP-2 favors cholesterol biogenesis. SREBP-1c-responsive targets include genes involved in fatty acid metabolism, such as fatty acid synthase (FAS) and acetyl-CoA carboxylase (ACC), and SREBP-2-responsive targets include genes involved in cholesterol metabolism, such as HMG-CoA synthase and HMG-CoA reductase (Moon et al., 2001). SREBPs are regulated by SREBP-cleavage-activating protein (SCAP), which is a sterol sensor. In response to low cellular cholesterol levels, SCAP transports SREBPs from the ER to the Golgi apparatus, where SREBP precursors are cleaved, releasing the transcriptionally active $\mathrm{N}$-terminus. When cholesterol is abundant in cells, the SREBP-SCAP complex is retained in the ER (Goldstein et al., 2006).

In the formation of macrophage-derived foam cells, the intracellular cholesterol accumulation is in part due to the defective suppression of SREBP-dependent gene expression and the failure to appropriately activate liver $X$ receptor (LXR)-mediated pathways. LXRs can modulate cholesterol efflux from macrophages into ApoA1 and high density lipoproteins (HDL) via the ABCA1 and ABCG1 pathways, although LXRs also induce the uptake of cholesterol esters from HDLs in primary human macrophages (Bultel et al., 2008). A recent study has demonstrated SREBP-1a is abundantly expressed in macrophages (Im et al., 2011). Interestingly, lipopolysaccharide (LPS), which was shown to increase de novo lipogenesis in macrophages in vivo (Posokhova et al., 2008), upregulates SREBP-1a mRNA and nuclear protein levels, and stimulates lipogenesis in wild-type but not SREBP-1a-deficient macrophages (Im et al., 2011).

\section{PPARs}

The PPARs are known to be crucial players in lipid and glucose homeostasis, and all three PPARs $(\alpha, \gamma$, and $\delta$ ) have been shown to exist in macrophage-derived foam cells (Rader and Puré, 2005). In mouse models, genetic deletion of macrophage PPAR $\gamma$ resulted in increased development of atherosclerosis (Chawla et al., 2001), whereas genetic deletion of PPAR $\alpha$ (Tordjman et al., 2001) and PPAR $\delta$ (Lee et al., 2003) led to reduced atherosclerosis. Similarly, PPAR $\alpha$ and PPAR $\gamma$ agonists have been shown to significantly reduce atherosclerotic lesions in mice. However, a synthetic PPAR $\delta$ agonist has little effect on atherosclerosis in mice ( $\mathrm{Li}$ et al., 2004), suggesting that PPARs may have diverse effects on the atherogenic processes. In macrophages, PPAR $\alpha$ also induces the expression of NPC1 and NPC2, which control the transport of free cholesterol from the lysosome to the plasma membrane (Chinetti-Gbaguidi et al., 2005), thus leading to increased cholesterol efflux. In a similar pathway, PPAR $\alpha$ and $\gamma$ induce ABCA1 expression (Chinetti et al., 2001), which also aids cholesterol efflux from macrophages and cholesterol reverse transport.

It has been demonstrated that in the macrophage-derived foam cells oxLDL activates PPAR $\alpha$ and $\gamma$ in a dosedependent manner (Taketa et al., 2008). PPAR $\gamma$ activation by oxLDL occurs via protein kinase $C$ (PKC) and might be a mechanism to regulate the expression of CD36. Interestingly, CD36 itself has been reported to activate PPAR $($ Lee et al., 2004), thus creating a positive feedback loop regulating this transcription factor. Moreover, the expression of VEGF, which is an angiogenic factor that promotes macrophage migration, is stimulated by oxLDL in a PPAR $\gamma$-dependent manner. Thus, further studies are required to better elucidate the complex role of PPARs in foam cell formation and atherogenesis.

\section{LIPID DROPLET ASSOCIATED PROTEINS}

LD-associated proteins are localized on the surface of LDs and are crucial for the formation and catabolism of intracellular LDs. Recently, it has been demonstrated that a special set of LD-associated proteins, the PAT family proteins, are important for the formation, growth, stabilization and functions of LDs in atherosclerotic foam cells (Paul et al., 2008a; Buers et al., 2011) (Fig. 2). The PAT family of proteins is named after its members perilipin (perilipin 1), adipose differentiation-related protein (ADRP, adipophilin, or perilipin 2), tail interacting protein of $47 \mathrm{kDa}$ (TIP47 or perilipin 3), S3-12 (perilipin 4) and LSDP5 (MLDP, OXPAT, or perilipin 5).

\section{ADRP}

ADRP is the most abundant and best characterized PAT protein in human and mouse macrophage-derived foam cells (Buechler et al., 2001; Larigauderie et al., 2004; Paul et al., 2008b). Interestingly, the expression of ADRP in the atherosclerosis-studded arteries of $A p o E$-deficient mice was approximately 3.5-fold higher than in the atherosclerosis-free arteries of wild-type mice (Paul et al., 2008b). Inactivation of the Adrp gene in ApoE-deficient mice significantly reduced the number of lipid droplets in the foam cells in atherosclerotic lesions and protected mice against atherosclerosis (Paul et al., 2008b). Moreover, overexpression of ADRP resulted in increased lipid droplet accumulation due to reduced cholesterol efflux from THP-1 macrophages (Larigauderie et al., 2004). In contrast, peritoneal macrophages isolated from 
Adrp -deficient mice demonstrated greater cholesterol efflux than those isolated from their wild-type littermates (Paul et al., 2008b). However, detailed studies to elucidate the mechanistic role of ADRP in the formation of macrophage-derived foam cells have not been performed, and it is possible that ADRP, as an LD-associated protein, could protect LDs against the hydrolysis of lipases. In HEK293 cells, overexpression of ADRP has been shown to correlate with decreased association of the major TG lipase named ATGL with LDs and increased TG accumulation as a result of inhibition of TG hydrolysis (Listenberger et al., 2007). ADRP overexpression also increased TG content in THP-1-derived macrophages by promoting the incorporation of fatty acyl-CoAs into TGs and inhibiting fatty acid oxidation (Larigauderie et al., 2006). Moreover, ADRP augments TNF $\alpha$, MCP-1, and IL-6 induction in acLDL-induced macrophages, suggesting that enhancing inflammation might be one role of ADRP in atherosclerosis (Chen et al., 2010). In addition, LPS and other Toll-like receptor (TLR) ligands, which have been previously shown to increase macrophage TG and CE accumulation (Tobias and Curtiss, 2005; Posokhova et al., 2008), increase the expression of ADRP as part of a coordinated change in macrophage physiology (Feingold et al., 2010).

\section{Perilipin}

Although perilipin has been detected in THP-1 cells and human monocyte-derived macrophages, its role in regulating foam cell formation is still controversial (Hofnagel et al., 2007). Perilipin was found to be expressed in foam cells adjacent to ruptured plaques but was absent in stable plaques of human atherosclerotic lesions (Faber et al., 2001). Because ADRP coats the surface of LDs of perilipin-null adipocytes (Tansey et al., 2001), it was predicted that in the absence of ADRP, perilipin could eventually take over its role in foam cells. However, perilipin was not detected in mouse lipid-laden macrophages that do not express ADRP (Paul et al., 2008b). In mice with both perilipin and Ldlr deficiency, the atherosclerotic lesion area moderately increased after 10 weeks of atherogenic diet but was significantly larger after 20 weeks (Langlois et al., 2011), strongly supporting an atheroprotective role of perilipin.

\section{TIP47/S-12/LSDP5}

The third member of the PAT family of proteins, TIP47, was described originally as a cytosolic protein that binds to the cytoplasmic domains of cation-dependent and cation-independent mannose-6-phosphate receptors (Buers et al., 2009). TIP47 is located on the LD surface of Hela and other cell types, including human THP-1 macrophages (Robenek et al., 2005a, 2005b), but TIP47 expression is not stimulated by oxLDL. However, knockdown of TIP47 by siRNA specifically reduced cellular TG content but not cholesterol content, indicating that TIP47 is mainly involved in TG accumulation (Gu et al., 2010). It has been demonstrated that TIP47 acts as a carrier protein for FFAs and is involved in foam cell formation (Buers et al., 2009). Using microarray technology, it has been shown that S3-12 and LSDP5 expression increases after oxLDL loading of THP-1 cells, but the TIP47 expression decreases (Li et al., 2010). However, the detailed role of TIP47, S3-12, and LSDP5 in foam cell formation has not been elucidated yet.

\section{CIDE proteins}

The cell death-inducing DFF45-like effector (CIDE) family, another LD-associated protein family, is comprised of three members: Cidea, Cideb, and Cidec, which is called Fsp27 in rodents (Gong et al., 2009). Among CIDE proteins, Cideb promotes the formation of TG-enriched VLDL particles and provides a molecular insight into VLDL lipidation and maturation in hepatocytes (Ye et al., 2009). Cideb deficiency results in lower plasma TGs and FA levels (Li and Li, 2007; Li et al., 2007), thereby suggesting a potential role for Cideb in the development of atherosclerosis. Microarray and qRT-PCR analysis showed that during the formation of THP-1-derived foam cells, Cideb mRNA levels were upregulated, which was also confirmed by analyzing their protein level ( $\mathrm{Li}$ et al., 2010).

Similarly, Fsp27-deficient mice have dramatically lower levels of serum TGs and much smaller lipid droplets in their white adipocytes (Nishino et al., 2008; Toh et al., 2008). Fsp27 deficiency results in higher lipolysis rates, especially for basal lipolysis, and leads to a reduction in fat accumulation (Nishino et al., 2008; Toh et al., 2008). Ectopic expression of Fsp27 in 3T3-L1 preadipocytes promotes the formation of significantly larger LDs, and enhances accumulation of total neutral lipids (Puri et al., 2007). Consistent with this observation, Cidec knockdown using RNA interference significantly stimulates lipolysis and results in the fragmentation of large storage lipid droplets to multiple small ones (Puri et al., 2007). Like perilipin, both Cidea and Cidec are regulated by PPAR $\gamma$ (Puri et al., 2008), and it is possible that the CIDE family proteins, similar to the PAT members, may play multiple roles in the formation of macrophage-derived foam cells.

Despite the emerging relevance of LD-associated proteins to atherogenesis, studies on their specific role in lipid metabolism in macrophage-derived foam cells are still at a very early stage. The variety of expression patterns and localization of the LD-associated proteins in LD-containing cells suggests multiple roles for these proteins in atherogenesis. Relevant questions to be addressed in the future are: (1) what are the roles of LD-associated proteins, other than ADRP, in foam cell formation? (2) how do the LD-associated proteins regulate LD metabolism in foam cells? and (3) could the LD-associated proteins be used as therapeutic targets to 
prevent foam cell formation and intervene with the process of atherogenesis?

\section{CONCLUSION}

In conclusion, the formation of macrophage-derived foam cells is a hallmark of atherosclerosis. During the development of atherosclerotic plaques, monocytes migrate into the arterial intima where they differentiate into macrophages and become lipid-overloaded by uptake of excess amounts of modified lipoproteins. Simultaneously, lipotoxicity is triggered by the elevated intracellular concentration of lipids, especially FC and FAs. During this process, transcription factors such as SREBPs and PPARs become activated to regulate lipid homeostasis. With the accumulation of excess lipids in the macrophages, a mass of LDs is formed in the cytoplasm leading to the formation of foam cells. It has now been established that LD-associated proteins, such as the PAT family and CIDE family proteins, are crucial for the formation and catabolism of intracellular LDs and also play multiple roles in the formation, growth and stabilization of LDs in atherosclerotic foam cells. Identification of novel factors and elucidation of their mechanistic roles in foam cell formation will help in the development of therapeutic interventions for atherosclerosis.

\section{ACKNOWLEDGEMENTS}

This work was supported by the Natural Science Foundation of China (Grant Nos. 81070248, 81070249, 31171132, and 81100612). The authors acknowledge invaluable discussions with members of their laboratories and colleagues in the field.

\section{ABBREVIATIONS}

ABCA1, ATP-binding cassette transporter A1; ACAT1, acyl-CoA:cholesterol acyltransferase 1; ACC, acetyl-CoA carboxylase; ADRP, adipose differentiation-related protein; bHLH-Zip, basic-helix-loop-helix-leucine zipper; CD36, cluster differentiation 36; $\mathrm{CE}$, cholesterol esters; CIDE, cell death-inducing DFF45-like effector; $E R$, endoplasmic reticulum; FA, fatty acids; FAS, fatty acid synthase; FC, free cholesterol; HDL, high-density lipoprotein; LAL, Iysosomal acid lipase; LD, lipid droplets; LPS, lipopolysaccharide; LXR, liver X receptor; $\mathrm{nCEH}$ neutral cholesterol ester hydrolase; oxLDL, oxidized low-density lipoprotein; PKC, protein kinase C; PPARs, peroxisome proliferator-activated receptors; RCT, reverse cholesterol transport; SCAP, SREBP-cleavage-activating protein; SR, scavenger receptors; SR-A, type A scavenger receptor; SREBP, sterol regulatory element-binding proteins; TG, triglycerides; TLR, Toll-like receptor; TIP47, tail interacting protein of $47 \mathrm{kDa}$

\section{REFERENCES}

Adorni, M.P., Zimetti, F., Billheimer, J.T., Wang, N., Rader, D.J., Phillips, M.C., and Rothblat, G.H. (2007). The roles of different pathways in the release of cholesterol from macrophages. J Lipid
Res 48, 2453-2462.

An, G., Wang, H., Tang, R., Yago, T., McDaniel, J.M., McGee, S., Huo, Y., and Xia, L. (2008). P-selectin glycoprotein ligand-1 is highly expressed on Ly-6Chi monocytes and a major determinant for Ly-6Chi monocyte recruitment to sites of atherosclerosis in mice. Circulation 117, 3227-3237.

Ashraf, M.Z., and Gupta, N. (2011). Scavenger receptors: Implications in atherothrombotic disorders. Int J Biochem Cell Biol 43, 697-700.

Borradaile, N.M., Han, X., Harp, J.D., Gale, S.E., Ory, D.S., and Schaffer, J.E. (2006). Disruption of endoplasmic reticulum structure and integrity in lipotoxic cell death. J Lipid Res 47, 2726-2737.

Brown, M.S., and Goldstein, J.L. (1997). The SREBP pathway: regulation of cholesterol metabolism by proteolysis of a membrane-bound transcription factor. Cell 89, 331-340.

Brown, M.S., and Goldstein, J.L. (1999). A proteolytic pathway that controls the cholesterol content of membranes, cells, and blood. Proc Natl Acad Sci U S A 96, 11041-11048.

Brunham, L.R., Singaraja, R.R., Duong, M., Timmins, J.M., Fievet, C., Bissada, N., Kang, M.H., Samra, A., Fruchart, J.C., McManus, B., et al. (2009). Tissue-specific roles of ABCA1 influence susceptibility to atherosclerosis. Arterioscler Thromb Vasc Biol 29, 548-554.

Buechler, C., Ritter, M., Duong, C.Q., Orso, E., Kapinsky, M., and Schmitz, G. (2001). Adipophilin is a sensitive marker for lipid loading in human blood monocytes. Biochim Biophys Acta 1532, 97-104.

Buers, I., Hofnagel, O., Ruebel, A., Severs, N.J., and Robenek, H. (2011). Lipid droplet associated proteins: an emerging role in atherogenesis. Histol Histopathol 26, 631-642.

Buers, I., Robenek, H., Lorkowski, S., Nitschke, Y., Severs, N.J., and Hofnagel, O. (2009). TIP47, a lipid cargo protein involved in macrophage triglyceride metabolism. Arterioscler Thromb Vasc Biol 29, 767-773.

Bultel, S., Helin, L., Clavey, V., Chinetti-Gbaguidi, G., Rigamonti, E., Colin, M., Fruchart, J.C., Staels, B., and Lestavel, S. (2008). Liver $X$ receptor activation induces the uptake of cholesteryl esters from high density lipoproteins in primary human macrophages. Arterioscler Thromb Vasc Biol 28, 2288-2295.

Burgess, B., Naus, K., Chan, J., Hirsch-Reinshagen, V., Tansley, G., Matzke, L., Chan, B., Wilkinson, A., Fan, J., Donkin, J., et al. (2008). Overexpression of human ABCG1 does not affect atherosclerosis in fat-fed ApoE-deficient mice. Arterioscler Thromb Vasc Biol 28, 1731-1737.

Chawla, A., Boisvert, W.A., Lee, C.H., Laffitte, B.A., Barak, Y., Joseph, S.B., Liao, D., Nagy, L., Edwards, P.A., Curtiss, L.K., et al. (2001). A PPAR gamma-LXR-ABCA1 pathway in macrophages is involved in cholesterol efflux and atherogenesis. Mol Cell 7, 161-171.

Chen, F.L., Yang, Z.H., Wang, X.C., Liu, Y., Yang, Y.H., Li, L.X., Liang, W.C., Zhou, W.B., and Hu, R.M. (2010). Adipophilin affects the expression of TNF-alpha, MCP-1, and IL-6 in THP-1 macrophages. Mol Cell Biochem 337, 193-199.

Chinetti, G., Lestavel, S., Bocher, V., Remaley, A.T., Neve, B., Torra, I.P., Teissier, E., Minnich, A., Jaye, M., Duverger, N., et al. (2001). PPAR-alpha and PPAR-gamma activators induce cholesterol removal from human macrophage foam cells through stimulation of the ABCA1 pathway. Nat Med 7, 53-58.

Chinetti-Gbaguidi, G., Rigamonti, E., Helin, L., Mutka, A.L., Lepore, 
M., Fruchart, J.C., Clavey, V., Ikonen, E., Lestavel, S., and Staels, B. (2005). Peroxisome proliferator-activated receptor alpha controls cellular cholesterol trafficking in macrophages. J Lipid Res 46 , 2717-2725.

Combadière, C., Potteaux, S., Rodero, M., Simon, T., Pezard, A., Esposito, B., Merval, R., Proudfoot, A., Tedgui, A., and Mallat, Z. (2008). Combined inhibition of CCL2, CX3CR1, and CCR5 abrogates Ly6C(hi) and Ly6C(lo) monocytosis and almost abolishes atherosclerosis in hypercholesterolemic mice. Circulation 117, 1649-1657.

Duewell, P., Kono, H., Rayner, K.J., Sirois, C.M., Vladimer, G., Bauernfeind, F.G., Abela, G.S., Franchi, L., Nuñez, G., Schnurr, M., et al. (2010). NLRP3 inflammasomes are required for atherogenesis and activated by cholesterol crystals. Nature 464, 1357-1361.

Faber, B.C., Cleutjens, K.B., Niessen, R.L., Aarts, P.L., Boon, W., Greenberg, A.S., Kitslaar, P.J., Tordoir, J.H., and Daemen, M.J. (2001). Identification of genes potentially involved in rupture of human atherosclerotic plaques. Circ Res 89, 547-554.

Fazio, S., Major, A.S., Swift, L.L., Gleaves, L.A., Accad, M., Linton, M.F., and Farese, R.V. Jr. (2001). Increased atherosclerosis in LDL receptor-null mice lacking ACAT1 in macrophages. J Clin Invest 107, 163-171.

Feingold, K.R., Kazemi, M.R., Magra, A.L., McDonald, C.M., Chui, L.G., Shigenaga, J.K., Patzek, S.M., Chan, Z.W., Londos, C., and Grunfeld, C. (2010). ADRP/ADFP and Mal1 expression are increased in macrophages treated with TLR agonists. Atherosclerosis $209,81-88$.

Ghosh, S., St Clair, R.W., and Rudel, L.L. (2003). Mobilization of cytoplasmic CE droplets by overexpression of human macrophage cholesteryl ester hydrolase. J Lipid Res 44, 1833-1840.

Ghosh, S., Zhao, B., Bie, J., and Song, J. (2010). Macrophage cholesteryl ester mobilization and atherosclerosis. Vascul Pharmacol $52,1-10$.

Glass, C.K., and Witztum, J.L. (2001). Atherosclerosis. the road ahead. Cell 104, 503-516.

Goldstein, J.L., DeBose-Boyd, R.A., and Brown, M.S. (2006). Protein sensors for membrane sterols. Cell 124, 35-46.

Gong, J., Sun, Z., and Li, P. (2009). CIDE proteins and metabolic disorders. Curr Opin Lipidol 20, 121-126.

Gordon, S., and Martinez, F.O. (2010). Alternative activation of macrophages: mechanism and functions. Immunity 32, 593-604.

Gu, J.Q., Wang, D.F., Yan, X.G., Zhong, W.L., Zhang, J., Fan, B., and Ikuyama, S. (2010). A Toll-like receptor 9-mediated pathway stimulates perilipin 3 (TIP47) expression and induces lipid accumulation in macrophages. Am J Physiol Endocrinol Metab 299, E593-E600.

Hofnagel, O., Buers, I., Schnoor, M., Lorkowski, S., and Robenek, H. (2007). Expression of perilipin isoforms in cell types involved in atherogenesis. Atherosclerosis 190, 14-15, author reply 16-17.

Im, S.S., Yousef, L., Blaschitz, C., Liu, J.Z., Edwards, R.A., Young, S.G., Raffatellu, M., and Osborne, T.F. (2011). Linking lipid metabolism to the innate immune response in macrophages through sterol regulatory element binding protein-1a. Cell Metab 13, 540-549.

Kadl, A., Meher, A.K., Sharma, P.R., Lee, M.Y., Doran, A.C., Johnstone, S.R., Elliott, M.R., Gruber, F., Han, J., Chen, W., et al. (2010). Identification of a novel macrophage phenotype that develops in response to atherogenic phospholipids via Nrf2. Circ Res 107, 737-746.

Kunjathoor, V.V., Febbraio, M., Podrez, E.A., Moore, K.J., Andersson,
L., Koehn, S., Rhee, J.S., Silverstein, R., Hoff, H.F., and Freeman, M.W. (2002). Scavenger receptors class A-I/II and CD36 are the principal receptors responsible for the uptake of modified low density lipoprotein leading to lipid loading in macrophages. J Biol Chem 277, 49982-49988.

Langlois, D., Forcheron, F., Li, J.Y., del Carmine, P., Neggazi, S., and Beylot, M. (2011). Increased atherosclerosis in mice deficient in perilipin1. Lipids Health Dis 10, 169.

Larigauderie, G., Cuaz-Pérolin, C., Younes, A.B., Furman, C., Lasselin, C., Copin, C., Jaye, M., Fruchart, J.C., and Rouis, M. (2006) Adipophilin increases triglyceride storage in human macrophages by stimulation of biosynthesis and inhibition of beta-oxidation. FEBS J 273, 3498-3510.

Larigauderie, G., Furman, C., Jaye, M., Lasselin, C., Copin, C., Fruchart, J.C., Castro, G., and Rouis, M. (2004). Adipophilin enhances lipid accumulation and prevents lipid efflux from THP-1 macrophages: potential role in atherogenesis. Arterioscler Thromb Vasc Biol 24, 504-510.

Lee, C.H., Chawla, A., Urbiztondo, N., Liao, D., Boisvert, W.A., Evans, R.M., and Curtiss, L.K. (2003). Transcriptional repression of atherogenic inflammation: modulation by PPARdelta. Science 302, 453-457.

Lee, K.J., Kim, H.A., Kim, P.H., Lee, H.S., Ma, K.R., Park, J.H., Kim, D.J., and Hahn, J.H. (2004). Ox-LDL suppresses PMA-induced MMP-9 expression and activity through CD36-mediated activation of PPAR-g. Exp Mol Med 36, 534-544.

Li, A.C., Binder, C.J., Gutierrez, A., Brown, K.K., Plotkin, C.R., Pattison, J.W., Valledor, A.F., Davis, R.A., Willson, T.M., Witztum, J.L., et al. (2004). Differential inhibition of macrophage foam-cell formation and atherosclerosis in mice by PPARalpha, beta/delta, and gamma. J Clin Invest 114, 1564-1576.

Li, H., Song, Y., Li, F., Zhang, L., Gu, Y., Zhang, L., Jiang, L., Dong, W., Ye, J., and Li, Q. (2010). Identification of lipid droplet-associated proteins in the formation of macrophage-derived foam cells using microarrays. Int J Mol Med 26, 231-239.

Li, J.Z., and Li, P. (2007). Cide proteins and the development of obesity. Novartis Found Symp 286, 155-159; discussion 159-163, 196-203.

Li, J.Z., Ye, J., Xue, B., Qi, J., Zhang, J., Zhou, Z., Li, Q., Wen, Z., and Li, P. (2007). Cideb regulates diet-induced obesity, liver steatosis, and insulin sensitivity by controlling lipogenesis and fatty acid oxidation. Diabetes 56, 2523-2532.

Listenberger, L.L., Ostermeyer-Fay, A.G., Goldberg, E.B., Brown, W.J., and Brown, D.A. (2007). Adipocyte differentiation-related protein reduces the lipid droplet association of adipose triglyceride lipase and slows triacylglycerol turnover. J Lipid Res 48, 2751-2761.

Makowski, L., Boord, J.B., Maeda, K., Babaev, V.R., Uysal, K.T., Morgan, M.A., Parker, R.A., Suttles, J., Fazio, S., Hotamisligil, G.S., et al. (2001). Lack of macrophage fatty-acid-binding protein aP2 protects mice deficient in apolipoprotein E against atherosclerosis. Nat Med 7, 699-705.

Manning-Tobin, J.J., Moore, K.J., Seimon, T.A., Bell, S.A., Sharuk, M., Alvarez-Leite, J.I., de Winther, M.P., Tabas, I., and Freeman, M.W. (2009). Loss of SR-A and CD36 activity reduces atherosclerotic lesion complexity without abrogating foam cell formation in hyperlipidemic mice. Arterioscler Thromb Vasc Biol 29, 19-26.

Martinez, F.O., Gordon, S., Locati, M., and Mantovani, A. (2006). Transcriptional profiling of the human monocyte-to-macrophage differentiation and polarization: new molecules and patterns of 
gene expression. J Immunol 177, 7303-7311.

Moon, Y.A., Shah, N.A., Mohapatra, S., Warrington, J.A., and Horton, J.D. (2001). Identification of a mammalian long chain fatty acyl elongase regulated by sterol regulatory element-binding proteins. J Biol Chem 276, 45358-45366.

Moore, K.J., Kunjathoor, V.V., Koehn, S.L., Manning, J.J., Tseng, A.A., Silver, J.M., McKee, M., and Freeman, M.W. (2005). Loss of receptor-mediated lipid uptake via scavenger receptor A or CD36 pathways does not ameliorate atherosclerosis in hyperlipidemic mice. J Clin Invest 115, 2192-2201.

Nishino, N., Tamori, Y., Tateya, S., Kawaguchi, T., Shibakusa, T., Mizunoya, W., Inoue, K., Kitazawa, R., Kitazawa, S., Matsuki, Y., et al. (2008). FSP27 contributes to efficient energy storage in murine white adipocytes by promoting the formation of unilocular lipid droplets. J Clin Invest 118, 2808-2821.

Ouimet, M., Franklin, V., Mak, E., Liao, X., Tabas, I., and Marcel, Y.L. (2011). Autophagy regulates cholesterol efflux from macrophage foam cells via lysosomal acid lipase. Cell Metab 13, 655-667.

Paul, A., Chan, L., and Bickel, P.E. (2008a). The PAT family of lipid droplet proteins in heart and vascular cells. Curr Hypertens Rep 10, 461-466.

Paul, A., Chang, B.H., Li, L., Yechoor, V.K., and Chan, L. (2008b) Deficiency of adipose differentiation-related protein impairs foam cell formation and protects against atherosclerosis. Circ Res 102, 1492-1501.

Pello, O.M., Silvestre, C., De Pizzol, M., and Andrés, V. (2011). A glimpse on the phenomenon of macrophage polarization during atherosclerosis. Immunobiology 216, 1172-1176.

Perrey, S., Legendre, C., Matsuura, A., Guffroy, C., Binet, J., Ohbayashi, S., Tanaka, T., Ortuno, J.C., Matsukura, T., Laugel, T., et al. (2001). Preferential pharmacological inhibition of macrophage ACAT increases plaque formation in mouse and rabbit models of atherogenesis. Atherosclerosis 155, 359-370.

Podrez, E.A., Febbraio, M., Sheibani, N., Schmitt, D., Silverstein, R.L., Hajjar, D.P., Cohen, P.A., Frazier, W.A., Hoff, H.F., and Hazen, S.L. (2000). Macrophage scavenger receptor CD36 is the major receptor for LDL modified by monocyte-generated reactive nitrogen species. J Clin Invest 105, 1095-1108.

Posokhova, E.N., Khoshchenko, O.M., Chasovskikh, M.I., Pivovarova, E.N., and Dushkin, M.I. (2008). Lipid synthesis in macrophages during inflammation in vivo: effect of agonists of peroxisome proliferator activated receptors alpha and gamma and of retinoid X receptors. Biochemistry (Mosc) 73, 296-304.

Puri, V., Konda, S., Ranjit, S., Aouadi, M., Chawla, A., Chouinard, M., Chakladar, A., and Czech, M.P. (2007). Fat-specific protein 27, a novel lipid droplet protein that enhances triglyceride storage. J Biol Chem 282, 34213-34218.

Puri, V., Ranjit, S., Konda, S., Nicoloro, S.M., Straubhaar, J., Chawla, A., Chouinard, M., Lin, C., Burkart, A., Corvera, S., et al. (2008). Cidea is associated with lipid droplets and insulin sensitivity in humans. Proc Natl Acad Sci U S A 105, 7833-7838.

Rader, D.J., and Puré, E. (2005). Lipoproteins, macrophage function, and atherosclerosis: beyond the foam cell? Cell Metab 1, 223-230.

Rios, F.J., Gidlund, M., and Jancar, S. (2011). Pivotal role for platelet-activating factor receptor in CD36 expression and oxLDL uptake by human monocytes/macrophages. Cell Physiol Biochem 27, 363-372

Robenek, H., Lorkowski, S., Schnoor, M., and Troyer, D. (2005a). Spatial integration of TIP47 and adipophilin in macrophage lipid bodies. J Biol Chem 280, 5789-5794.

Robenek, H., Robenek, M.J., and Troyer, D. (2005b). PAT family proteins pervade lipid droplet cores. J Lipid Res 46, 1331-1338.

Siegel-Axel, D., Daub, K., Seizer, P., Lindemann, S., and Gawaz, M. (2008). Platelet lipoprotein interplay: trigger of foam cell formation and driver of atherosclerosis. Cardiovasc Res 78, 8-17.

Takahashi, K., Takeya, M., and Sakashita, N. (2002). Multifunctional roles of macrophages in the development and progression of atherosclerosis in humans and experimental animals. Med Electron Microsc 35, 179-203.

Taketa, K., Matsumura, T., Yano, M., Ishii, N., Senokuchi, T., Motoshima, H., Murata, Y., Kim-Mitsuyama, S., Kawada, T., Itabe, H., et al. (2008). Oxidized low density lipoprotein activates peroxisome proliferator-activated receptor-alpha (PPARalpha) and PPARgamma through MAPK-dependent COX-2 expression in macrophages. J Biol Chem 283, 9852-9862.

Tansey, J.T., Sztalryd, C., Gruia-Gray, J., Roush, D.L., Zee, J.V., Gavrilova, O., Reitman, M.L., Deng, C.X., Li, C., Kimmel, A.R., et al. (2001). Perilipin ablation results in a lean mouse with aberrant adipocyte lipolysis, enhanced leptin production, and resistance to diet-induced obesity. Proc Natl Acad Sci U S A 98, 6494-6499.

Tobias, P., and Curtiss, L.K. (2005). Thematic review series: The immune system and atherogenesis. Paying the price for pathogen protection: toll receptors in atherogenesis. J Lipid Res 46, 404-411.

Toh, S.Y., Gong, J., Du, G., Li, J.Z., Yang, S., Ye, J., Yao, H., Zhang, Y., Xue, B., Li, Q., et al. (2008). Up-regulation of mitochondrial activity and acquirement of brown adipose tissue-like property in the white adipose tissue of fsp27 deficient mice. PLoS One 3, e2890.

Tordjman, K., Bernal-Mizrachi, C., Zemany, L., Weng, S., Feng, C., Zhang, F., Leone, T.C., Coleman, T., Kelly, D.P., and Semenkovich, C.F. (2001). PPARalpha deficiency reduces insulin resistance and atherosclerosis in apoE-null mice. J Clin Invest 107, 1025-1034.

Trigatti, B., Rayburn, H., Viñals, M., Braun, A., Miettinen, H., Penman, M., Hertz, M., Schrenzel, M., Amigo, L., Rigotti, A., et al. (1999). Influence of the high density lipoprotein receptor SR-BI on reproductive and cardiovascular pathophysiology. Proc Natl Acad Sci U S A 96, 9322-9327.

Ye, J., Li, J.Z., Liu, Y., Li, X., Yang, T., Ma, X., Li, Q., Yao, Z., and Li, P. (2009). Cideb, an ER- and lipid droplet-associated protein, mediates VLDL lipidation and maturation by interacting with apolipoprotein B. Cell Metab 9, 177-190.

Yvan-Charvet, L., Ranalletta, M., Wang, N., Han, S., Terasaka, N., Li, R., Welch, C., and Tall, A.R. (2007). Combined deficiency of $A B C A 1$ and ABCG1 promotes foam cell accumulation and accelerates atherosclerosis in mice. J Clin Invest 117, 3900-3908.

Zhao, B., Song, J., Chow, W.N., St Clair, R.W., Rudel, L.L., and Ghosh, S. (2007). Macrophage-specific transgenic expression of cholesteryl ester hydrolase significantly reduces atherosclerosis and lesion necrosis in Ldlr mice. J Clin Invest 117, 2983-2992.

Zhao, Y., Pennings, M., Vrins, C.L., Calpe-Berdiel, L., Hoekstra, M., Kruijt, J.K., Ottenhoff, R., Hildebrand, R.B., van der Sluis, R., Jessup, W., et al. (2011). Hypocholesterolemia, foam cell accumulation, but no atherosclerosis in mice lacking $A B C$-transporter $\mathrm{A} 1$ and scavenger receptor $\mathrm{BI}$. Atherosclerosis 218, 314-322.

Zhou, X., He, W., Huang, Z., Gotto, A.M. Jr, Hajjar, D.P., and Han, J. (2008). Genetic deletion of low density lipoprotein receptor impairs sterol-induced mouse macrophage ABCA1 expression. A new SREBP1-dependent mechanism. J Biol Chem 283, 2129-2138. 